\title{
Elaboração de Informes Técnicos sobre o uso de medicamentos na COVID-19: um trabalho colaborativo de Centros de Informações sobre Medicamentos do Brasil
}

\author{
Elaboration of Technical Reports on the use of medicines in the \\ COVID-19: collaborative work by Drug Information Centers from Brazil
}

\begin{abstract}
Maria Fernanda Barros de Oliveira Brandão' iD

Luiz Eduardo Oliveira Matos"

Luise de Paula Soares"II ip

Isabel Dielle Sousa Lima Pio"II

Mirian Parente Monteirolv iD

Ana Claúdia de Brito Passos ${ }^{\mathrm{IV}}$ iD

Taís Cristina Unfer" iD

Deuzilane Muniz Nunes ${ }^{11, * *}$ (iD)

I Centro de Informações sobre Medicamentos (CIM), Conselho

Introdução: Há uma urgência em se ter medicamentos capazes de tratar a COVID-19, mas existe ainda carência de estudos e dificuldades no acesso a informações seguras. Os Centros de Informações sobre Medicamentos (CIM) atuam na produção e difusão de informações de qualidade, baseadas nas melhores evidências científicas. Objetivo: Descrever a experiência colaborativa da elaboração de notas técnicas (NT) e de alerta de medicamento (AM) sobre a utilização de medicamentos na COVID-19, realizada por membros de CIM do Brasil. Método: Trata-se de um relato de experiência do tipo descritivo e analítico, com abordagem qualitativa. Foi analisado inicialmente o perfil dos CIM participantes e de seus profissionais envolvidos, e então apresentado o método adotado para a condução do trabalho, que foi realizado entre março a junho de 2020. Resultados: Participaram quatro CIM do Nordeste brasileiro: o da Universidade Federal do Vale do São Francisco, o da Universidade Federal de Sergipe, campus Lagarto, o da Universidade Federal do Ceará e o CIM do Conselho Regional de Farmácia da Bahia. As etapas do processo de desenvolvimento dos documentos técnicos foram: 1) Definição dos objetivos; 2) Seleção dos temas; 3) Busca de informação; 4) Processo de construção e descrição; 5) Revisão e 6) Publicização. Foram produzidas quatro NT e um AM. Conclusões: A experiência deste trabalho colaborativo demonstra que os CIM estão exercendo relevante papel no combate a pandemia pelo novo coronavírus e a infodemia, promovendo informação de qualidade, baseada nas melhores evidências.
\end{abstract} Regional de Farmácia do Estado da Bahia (CRF-BA), Salvador, BA, Brasil

" Centro de Informações sobre Medicamentos (CIM), Departamento de Farmácia de Lagarto (DFAL), Universidade Federal de Sergipe, campus universitário Professor Antônio Garcia Filho (UFS-Lag), Lagarto, SE, Brasil

III Centro de Informações sobre Medicamentos (CIM), Faculdade de Farmácia, Universidade Federal do Vale do São Francisco (UNIVASF), Petrolina, PE, Brasil

iv Centro de Informação sobre Medicamentos (CIM), Departamento de Farmácia, Universidade Federal do Ceará (UFC), Fortaleza, CE, Brasil

* E-mail: deuzilane.univasf@gmail.com

Recebido: 10 jul 2020

Aprovado: 27 jul 2020

\section{RESUMO}

PALAVRAS-CHAVE: Infecções por Coronavírus; COVID-19; Medicina Baseada em Evidência; Serviços de Informação sobre Medicamentos

\section{ABSTRACT}

Introduction: There is an urgency to have drugs capable of treating COVID-19. However, there are still shortages of studies and difficulties in accessing secure information. Drug Information Centers (DIC) operate in the production and dissemination of quality, objective, and timely information, based on the best scientific evidence. Objective: To describe the collaborative experience of preparing Technical Notes (TN) and Drugs Alerts (DA) on the use of medicines at COVID-19, carried out by members of DICs in Brazil. Method: This is an experience report of a descriptive and analytical type, with a qualitative approach. The profile of the participating DICs and their professionals were initially analyzed, and then the method adopted for conducting the work from March to June 2020 was presented. Results: Four DICs from the Northeast of Brazil participated in this experience, that of the Federal University of Vale São Francisco, the Federal University of Sergipe Lagarto campus, the Federal University of Ceara and the DIC of the Regional Pharmacy Council of Bahia. The stages of the TN development process were: 1) Definition of the TN objectives; 2) Selection of themes; 3) Search for information; 4) Construction process and description of the TNs; 5) Review and 6) Publication of TNs. Four TNs were 
produced, on antihypertensive and non-steroidal anti-inflammatory drugs; hydroxychloroquine or chloroquine; ivermectin and, vitamin D, in addition, a DA about ivermectin and your potential to cause neurotoxicity. Conclusions: The experience of this collaborative work demonstrates that DICs are playing an important role in combating the pandemic by the new coronavirus and infodemic, promoting quality information, based on the best evidence.

KEYWORDS: Coronavirus Infections; COVID-19; Evidence-Based Medicine; Drug Information Services

\section{INTRODUÇÃO}

Uma pneumonia de causa desconhecida, detectada em Wuhan, China, foi relatada pela primeira vez em 31 de dezembro de 2019. Com sua disseminação por todos os continentes, em menos de três meses essa infecção causada pelo novo coronavírus teve seu status atualizado de surto para pandemia'.

No contexto de emergências de saúde pública, como é uma pandemia, uma das atividades científicas mais complexas é a avaliação de medicamentos candidatos a tratamento, quanto à eficácia e à segurança, com benefícios se sobrepondo aos riscos potenciais aos pacientes ${ }^{2}$. Por ser uma nova doença, pouco se conhece sobre a fisiopatologia e os tratamentos da COVID-19, o que traz incertezas quanto às abordagens empregadas. Além disso, a urgência em encontrar medicamentos efetivos torna-se ainda maior pelas complicações relacionadas à doença e ao risco de morte ${ }^{3}$.

Em todo o mundo, estudos têm sido realizados pela comunidade científica, porém nem sempre os ensaios clínicos incluem um grupo controle e testes em amostra de pacientes representativa da população. Entre os estudos mais promissores estão o ensaio clínico RECOVERY ${ }^{4}$, com dexametasona, que apresentou em seus resultados preliminares a redução de mortes em pacientes hospitalizados em estado crítico e o estudo NIAID-ACTT-1, que observou a aceleração da recuperação em pacientes com COVID-19 hospitalizados em estado grave, que utilizaram remdesivir ${ }^{5}$.

Diversos medicamentos que demonstram algum potencial para prevenir ou tratar a COVID-19 não possuem ainda evidências científicas conclusivas, não sendo possível afirmar que tragam mais benefícios do que riscos à saúde do paciente ${ }^{6}$. Ainda assim, emerge a utilização empírica e compassiva de fármacos, como exemplo a ivermectina e a hidroxicloroquina ${ }^{7}$. Contudo, as hipóteses terapêuticas, apoiam-se em pesquisas in vitro ${ }^{8,9}$, estudos ainda não publicados, em formato preprints ${ }^{4,5}$, e observacionais, realizados em epidemias anteriores ${ }^{10,11}$. Essa situação de grande incerteza tem gerado diferentes condutas entre especialistas sobre o tratamento de pacientes em diferentes estágios da doença ${ }^{6}$.

A apresentação e a disseminação acelerada de estudos, em fase inicial, in vitro, sem interpretação clara dos resultados, aumentam a pressão sobre profissionais de saúde e gestores de políticas públicas para prescrever ou selecionar esses medicamentos $^{3}$. Ademais, a exacerbada divulgação de informações estimula a busca por terapias não aprovadas ${ }^{9}$. Este cenário colabora para a utilização inadequada de medicamentos, trazendo problemas de ordem individual, como a ocorrência de reações adversas $^{12,13}$ e problemas com impacto coletivo, como desabastecimento nas farmácias, resultando em restrições regulatórias para dispensação $0^{14,15}$.

Os Centros e Serviços de Informações sobre Medicamentos (CIM/SIM) são instrumentos de apoio para: promoção da saúde; práticas seguras e racionais relacionadas ao uso de medicamentos; fortalecimento da gestão e das ações e serviços de saúde nos seus diferentes níveis ${ }^{16}$. Em alguns países, os CIM/SIM cooperam em redes em nível nacional, como no Peru ${ }^{17}$, Argentina ${ }^{18} \mathrm{e}$ Brasil ${ }^{19}$; e em âmbito internacional, como a rede UKMi no Reino Unido $^{20}$ e a Rede de Centros de Informações de Medicamentos da América Latina e do Caribe (Red CIMLAC) ${ }^{21}$.

No Brasil, existem diversos diversos CIM/SIM que atuam produzindo informações técnico-científicas, de forma objetiva, oportuna e atendendo às necessidades específicas com base nas melhores evidências disponíveis, seja por meio da Rede Brasileira ${ }^{19}$ ou por colaboração externa à rede. Estes serviços fornecem suporte à tomada de decisão clínica ao responder questionamentos de solicitantes (informações passivas ou reativas), bem como ao produzir espontaneamente materiais informativos, como notas técnicas (NT), alertas de medicamentos (AM), boletins, entre outros tipos de materiais (informações ativas ou proativas) ${ }^{22}$.

Entende-se por NT a documentação formal expedida por uma instituição ou um conjunto destas, para manifestar seu entendimento sobre determinado assunto. Serve para registrar esclarecimentos de dúvidas, sugestões e pontos de vista de natureza técnica. Deve ser elaborada com linguagem formal, por especialistas na matéria mediante análise completa do contexto, com histórico ou fundamento legal, baseados em informações relevantes ${ }^{23}$. Por sua vez, os $A M$ constituem-se numa ação de notificar uma audiência maior que a dos detentores iniciais da informação, uma suspeita de associação entre um medicamento e uma reação adversa ${ }^{24}$. Esses documentos devem ser informativos, impessoais e objetivos ${ }^{25}$.

Nesse prisma, pretende-se neste trabalho descrever a experiência colaborativa da elaboração de NT e AM sobre a utilização de medicamentos na COVID-19, realizada por membros de CIM do Brasil, como forma de subsidiar a tomada de decisão dos gestores, prescritores e outros profissionais de saúde.

\section{MÉTODO}

Trata-se de um relato de experiência do tipo descritivo e analítico, com abordagem qualitativa, sobre o trabalho integrado 
de CIM brasileiros para elaboração de NT e de um alerta sobre medicamentos durante a pandemia do novo coronavírus.

Inicialmente analisou-se o perfil dos CIM participantes e de seus profissionais envolvidos nesta vivência. Em seguida, foi apresentado o método adotado para a condução do trabalho colaborativo de março a junho de 2020, com descrição do perfil das informações já publicadas.

A integração dos colaboradores ocorreu a partir de um grupo de aplicativo de mensagens instantâneas composto por 50 farmacêuticos de 27 equipes de CIM/SIM integrantes da Rede Brasileira de Centros e Serviços de Informação sobre Medicamentos (Rebracim) e de serviços independentes, não vinculados à rede. 0 trabalho colaborativo foi motivado pela pandemia do novo coronavírus e pela necessidade de informações de qualidade. Partiu, então, da disponibilidade de alguns CIM, participantes do grupo, com o objetivo de contribuir na disseminação de informações confiáveis e seguras, com base nas melhores evidências científicas, que pudessem subsidiar a tomada de decisão de gestores e profissionais de saúde.

Em março de 2020, foi firmada uma parceria entre os serviços para a cooperação técnica, de forma totalmente virtual. Seis CIM iniciaram o trabalho integrado, mas dois deles não conseguiram permanecer, findando com a participação de quatro CIM do Nordeste brasileiro: da Universidade Federal do Vale do São Francisco (CIM-Univasf), da Universidade Federal de Sergipe Campus Lagarto (CIMUFS-Lag), da Universidade Federal do Ceará (CIM-UFC) e do Conselho Regional de Farmácia da Bahia (CIM-CRF-BA).

Para edição e compartilhamento dos documentos foi escolhida a ferramenta Drive ${ }^{\circledR}$ (Google products), devido à possibilidade de armazenamento e sincronização de arquivos e inúmeras possibilidades para o trabalho colaborativo a distância. Foi analisada, através do Google Analytics $®$, a quantidade de acessos para cada documento (NT e AM) publicado na íntegra.

\section{RESULTADOS}

Os CIM participantes estão integrados a universidades públicas federais (CIM-UFC, CIM-Univasf e CIMUFS-Lag) e ao conselho de classe farmacêutico do estado da Bahia (CIM-CRF-BA). Três desses centros são vinculados à Rebracim (CIM-UFC, CIM-Univasf e CIM-CRF-BA), sendo dois destes (CIM-UFC e CIM-CRF-BA) parte da representação brasileira na Red CIMLAC. Fizeram parte da equipe técnica seis farmacêuticas (com titulação máxima de especialista a doutor), com colaboração de seis discentes graduandos em farmácia, estagiários dos CIM participantes.

As etapas do processo de trabalho inicialmente definidas para a elaboração das NT envolveram: definição dos objetivos, seleção dos temas, busca de informação, processo de construção e descrição, revisão pelo grupo avaliador e publicização do produto - NT (Figura).

\section{Definição dos objetivos}

A importância deste momento é tornar claros os objetivos, para que o produto final não se transforme apenas em mais um material informativo. Além disso, um instrumento desenvolvido por CIM exige atenção e rigor na sua elaboração para garantir a responsabilidade com a promoção do Uso Racional de Medicamentos (URM) através da produção e da difusão de informação imparcial, bem referenciada e criticamente avaliada ${ }^{22}$.

\section{Seleção dos temas}

Os temas foram selecionados com base na natureza das solicitações de informações enviadas aos CIM participantes deste trabalho e das informações veiculadas pelas mídias sociais, com notícias falsas ou distorcidas, sem uma base científica sólida, sobre o uso de medicamentos na COVID-19. Foram, então, elencadas, para a elaboração das NT, as demandas mais urgentes de informação técnica (Quadro) (Figura).

\section{Busca de informação}

Realizou-se levantamento bibliográfico por meio de acesso a fontes primárias (revistas científicas tais como The Lancet; British Medical Journal etc.); fontes secundárias (PubMed, Biblioteca Cochrane, SciELO, Web of Science), materiais do Ministério da Saúde do Brasil, da Organização Mundial da Saúde (OMS), da Organização Pan-Americana da Saúde (OPAS), da Fundação Oswaldo Cruz (Fiocruz) e outras fontes de informação técnica, como em sites específicos de informações sobre medicamentos, como o do Conselho Federal de Farmácia, Micromedex Solutions ${ }^{\circledR}$ e Prescrire.

Quadro. Descrição dos temas e datas de publicação das notas técnicas, realizadas por meio do trabalho colaborativo de quatro Centros de Informações sobre Medicamentos (CIM) brasileiros, de março a junho de 2020.

\begin{tabular}{|c|c|c|}
\hline Número & Título & Data de Publicação \\
\hline $01 / 2020$ & $\begin{array}{c}\text { Ausência de evidências científicas que relacionem o agravamento da infecção causada pelo } \\
\text { novo coronavírus (SARS-CoV-2) e a utilização de alguns medicamentos anti-hipertensivos e anti- } \\
\text { inflamatórios não esteroidais (AINEs) }\end{array}$ & 17/março/2020 \\
\hline $02 / 2020$ & $\begin{array}{c}\text { Evidências científicas frágeis sobre a utilização do medicamento hidroxicloroquina ou cloroquina no } \\
\text { tratamento e/ou prevenção de infecção pelo novo coronavírus (SARS-CoV-2) }\end{array}$ & 07/abril/2020 \\
\hline $03 / 2020$ & $\begin{array}{l}\text { Evidências científicas sobre a utilização do medicamento ivermectina para tratamento de infecção } \\
\text { pelo novo coronavírus (SARS-CoV-2) }\end{array}$ & 20/abril/2020 \\
\hline $04 / 2020$ & $\begin{array}{c}\text { Análise de evidências científicas sobre a suplementação de vitamina D (colecalciferol) como } \\
\text { prevenção e tratamento da COVID-19 }\end{array}$ & 08/maio/2020 \\
\hline
\end{tabular}

Fonte: Elaborado pelos autores, 2020. 


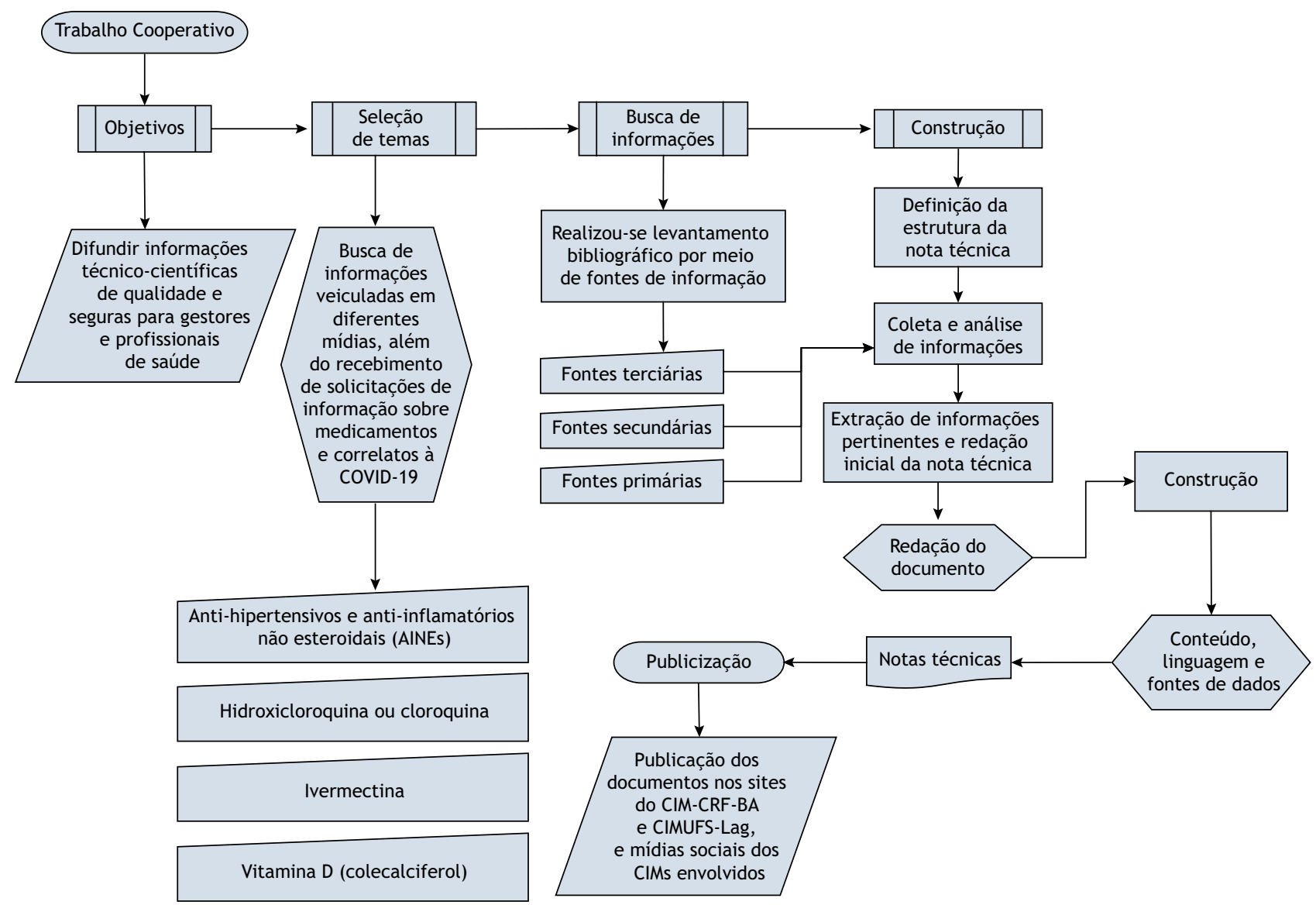

Fonte: Elaborada pelos autores, 2020.

Figura. Fluxograma do processo de trabalho cooperativo de quatro Centros de Informações sobre Medicamentos (CIM) brasileiros para desenvolvimento de notas técnicas sobre utilização de medicamentos na COVID-19.

\section{Processo de construção e descrição}

O primeiro passo consistiu em definir a estruturação do conteúdo das NT pela equipe. A versão inicial dos documentos era elaborada pelos estagiários, sob orientação constante da equipe técnica. Assim, realizou-se uma síntese das principais informações, com a contextualização do problema, a descrição das evidências disponíveis e a apresentação dos resultados e das recomendações.

Para a categorização dos artigos que foram utilizados na elaboração das NT, utilizou-se a classificação por tipo de delineamentos da pesquisa ${ }^{30}$. Foram utilizadas em média 9,5 (variando de 6 a 12) referências para o desenvolvimento de cada uma das NT, sendo: protocolos de tratamento $(n=1)$, notas informativas e/ou técnicas e posicionamentos de agências reguladoras nacional e internacionais $(n=9)$, editoriais, consensos e opiniões de especialistas $(n=4)$, ensaios clínicos não randomizados $(n=1)$, estudos observacionais $(n=1)$, revisões de literatura ou sistemática $(n=8)$, estudos in vitro $(n=6)$, relatos de caso ou série de casos $(n=3)$, além de artigos em formato preprints $(n=2)$, materiais técnicos e informativos disponibilizados no site do Conselho Federal de Farmácia $(n=1)$, Prescrire $(n=1)$, Formulário Terapêutico Nacional e Micromedex Solutions $₫(n=1)$.

\section{Grupo avaliador}

Para a avaliação inicial, uma versão de cada NT era disponibilizada em documento compartilhado para edição online. A equipe técnica discutia as evidências e corrigia o texto proposto até esgotar todas as dúvidas, chegando ao consenso da redação final.

\section{Publicização}

Após elaboração e revisão, as NT foram disponibilizadas na íntegra no site do CRF-BA e divulgadas no site do CIMUFS-Lag e nas mídias sociais dos CIM envolvidos. De acordo com dados fornecidos pelo Google Analytics $\circledast$, até o dia 9 de julho de 2020, as NT publicadas alcançaram 8.369 acessos no site do CRF-BA. A NT mais acessada foi sobre a ivermectina $(n=5.672,67,8 \%)$, seguida da hidroxicloroquina e cloroquina $(16,3 \%)$, vitamina $D$ $(9,1 \%)$ e AINEs $(6,8 \%)$.

Após a elaboração das NT surgiu uma demanda de informação relacionada ao risco neurotóxico associado ao uso de ivermectina na COVID-19. A equipe entendeu que a informação precisava ser amplamente divulgada também para a população em geral, não só aos profissionais de saúde e aos gestores. Diante disso, foi elaborado um documento em formato de AM seguindo as etapas do processo de desenvolvimento das NT a partir da 
busca de informação, incluindo o processo de construção e descrição, revisão pelo grupo avaliador e publicização do alerta $n^{\circ} 01 / 2020^{31}$, com tema: "Risco de neurotoxicidade causada pela ivermectina no tratamento da COVID-19". Este alerta foi divulgado no site do CRF-BA e do CIM/Univasf em 11 de junho de 2020, com o total de 6.355 acessos até o dia 09 de julho de 2020 . No site do CIM/Univasf, atingiu a maior porcentagem de acesso entre todas as páginas no período $(84,9 \%)$.

\section{DISCUSSÃO}

A elaboração das NT e do AM na COVID-19 contribui como ferramenta para o acesso a informações confiáveis aos profissionais de saúde, gestores e ainda à população em geral, em meio a avalanche de informações sem solidez, vivenciada junto a pandemia do novo coronavírus.

Não é a primeira vez que a humanidade sofre com uma pandemia. Pode ser citada a peste negra ${ }^{32}$, a infecção respiratória causada por um vírus da família Orthomyxoviridiae e manifestações de influenza que ocorreram no século XX. É possível ainda falar de outras epidemias provocadas por vírus da família Coronaviridae anteriores à COVID-19, como a epidemia de síndrome respiratória aguda grave (sigla em inglês: $S A R S)^{33}$ e a síndrome respiratória do Oriente Médio (sigla em inglês: MERS-CoV), que gerou uma epidemia que afetou vários países árabes e europeus $^{34}$. Esses dados permitem inferir que a convivência com as tragédias pandêmicas ou epidêmicas marcam a história evolutiva da humanidade.

Na atual pandemia, a urgência em produzir novos dados vêm contribuindo para um menor rigor e qualidade da informação, reduzindo o cuidado na análise das evidências, implicando na segurança do paciente e dos serviços de saúde. A baixa qualidade de informações em saúde foi descrita numa revisão sistemática de referências disponibilizadas na internet (incluindo 153 estudos transversais e 11.785 websites), sugerindo uma grande lacuna nas informações baseadas em evidências disponibilizadas ${ }^{35}$.

Essa situação se constitui em risco de saúde pública, sendo denominada tecnicamente como infodemia, uma situação em que o excesso de informações, precisas e imprecisas, dificultam o acesso a fontes idôneas e orientações confiáveis ${ }^{36}$. Na era da informação, esse fenômeno é amplificado pelas redes sociais e se propaga mais rapidamente ${ }^{37}$, podendo ser absorvida facilmente pelas pessoas, afetando seu comportamento e possivelmente elevando o risco de exposição ao vírus. Diante de tantas incertezas, a ampla divulgação de falsas notícias tem tornado a prática da educação em saúde um dos maiores desafios atuais ${ }^{38}$.

Assim, o fornecimento de informações baseadas em evidências para o público é uma das estratégias para mitigar a disseminação de notícias falsas ${ }^{37}$. Gerar conteúdo independente sobre medicamento que seja acessível a leigos ${ }^{24}$, aumentar a colaboração com jornalistas para minimizar erros de comunicação na tradução dos estudos e embasar a tomada de decisão dos profissionais de saúde são estratégias para mitigar a desinformação e suas consequências ${ }^{36}$.
Na elaboração das NT e do AM, a seleção dos estudos consistiu em uma etapa crucial. A carência de ensaios clínicos randomizados deram espaço para as notas informativas e/ou técnicas e posicionamentos de agências reguladoras nacional e internacionais, revisões de literatura e até estudos in vitro. Percebe-se ainda a presença de preprints, artigos disponibilizados antes da avaliação por pares. A elevada heterogeneidade e o baixo rigor metodológico dos estudos denotam o já referido incipiente controle de qualidade das publicações, característica da infodemia do período de pandemia.

Para a elaboração das NT e do AM foi realizada extensa análise e discussão crítica de toda a literatura disponível, a fim de que fossem produzidos com grande rigor técnico/científico e isentos de conflitos de interesse. É importante destacar ainda a capacidade técnica da equipe dos CIM, tendo em vista a formação e atuação dos profissionais, bem como a vinculação dos mesmos com universidades públicas federais, o que justifica a participação efetiva de estagiários e demonstra a importância do processo de ensino-aprendizagem na promoção de informações em saúde.

No contexto da publicização percebeu-se que o trabalho de divulgação colaborativo em todas as redes sociais dos CIM, os quais utilizam em suas rotinas de trabalho as novas tecnologias de informação, culminaram no grande alcance das NT e do AM. Essas ferramentas ajudam na promoção das informações aos usuários dos serviços, aos profissionais e à sociedade em geral, a partir de ferramentas práticas, rápidas e eficazes ${ }^{22}$.

Analisando os acessos virtuais de cada NT, verificou-se que a mais acessada foi a que tratou sobre a ivermectina, cuja recomendação foi de não se usar este medicamento no tratamento ou prevenção da COVID-19, uma vez que não há evidências científicas consistentes ${ }^{28}$, corroborando com outros informes técni$\cos ^{39,40,41}$. Além disso, a Sociedade Brasileira de Farmácia Clínica ${ }^{42}$ posicionou-se alertando para que a população e os profissionais da saúde sejam informados sobre a ausência de trabalhos em humanos e de que o efeito da ivermectina em vírus foi observado apenas in vitro. Assim, o uso de ivermectina para tratar COVID-19 é off label.

O tema ivermectina na COVID-19 ganhou destaque ainda devido aos seus riscos neurotóxicos, fato que levou os CIM a elaborarem o primeiro alerta ${ }^{31}$ com este foco, que teve maior acesso que a própria NT sobre ivermectina ${ }^{28}$. Outros informes incluindo dados sobre os efeitos potenciais de neurotoxicidade da ivermectina em pessoas com infecção pelo novo coronavírus foram emitidos em âmbito nacional e internacional após o alerta deste grupo $\mathrm{o}^{40,43}$.

Apesar dos argumentos contrários e que delineiam a preocupação quanto ao uso da ivermectina para tratar a COVID-19, esta vem sendo largamente utilizada em alguns países da América Latina, como Peru e Bolívia ${ }^{44,45}$. Além disso já se encontram registros sobre a disponibilização da ivermectina por redes públicas e de seguradoras de saúde privadas em diversas cidades brasileiras para prevenção e/ou tratamento da COVID-1946,47,48,49. O medicamento vem sendo motivo de ampla discussão no contexto do tratamento precoce da COVID-1944,45,46,47,48,49. 
A NT sobre a hidroxicloroquina e cloroquina, na COVID-1927, foi a segunda mais acessada. Sobre este tema houve diversas notas e informes em geral. Elas apresentaram pontos de análise comuns aos inseridos na NT deste grupo de CIM, reiterando as limitações metodológicas dos artigos encontrados e as fracas evidências científicas acerca da eficácia e da segurança dos medicamentos para embasar tomada de decisões ${ }^{50,51,52,53,54,55,56,57}$.

As NT com menos acessos foram sobre a vitamina $D^{29}$ e a utilização de medicamentos anti-hipertensivos e anti-inflamatórios não esteroidais (AINEs) ${ }^{26}$. Esta última corrobora com nota de esclarecimento da Sociedade Brasileira de Cardiologia ${ }^{58}$ e recomendações clínicas do Ministério da Saúde do Chile ${ }^{59,60}$, antes mesmo do posicionamento da OMS a respeito do assunto ${ }^{61}$.

Em relação à vitamina $D$ foram identificadas duas outras NT, uma do Conselho Regional de Medicina do Estado do Rio Grande do $\mathrm{Sul}^{62}$ e outra do Ministério da Saúde ${ }^{63}$, além de outras duas notas de esclarecimento ${ }^{64,65}$. Todas apontaram a não identificação de evidências conclusivas em relação à eficácia da utilização de vitamina $D$, na prevenção ou no tratamento de pessoas com COVID19 , corroborando com a NT elaborada por esta equipe ${ }^{62,63,64,65}$.

O caráter instrutivo das NT pode ser exemplificado na expedição da recomendação ${ }^{\circ}$ 11/2020 do Ministério Público do Pará, por meio da Promotoria de Justiça de Santarém ${ }^{66}$ que se fundamentou, entre outros documentos, na NT $n^{\circ} 3$, sobre utilização de ivermectina para tratamento da COVID-1928. Nesta normativa, a promotoria de Santarém recomenda aos serviços de saúde locais, entre outras coisas, a obtenção de consentimento escrito do usuário para o uso de farmacoterapias com hidroxicloroquina, ivermectina e corticosteroides, assim como se abster do uso em caso de negativa do paciente ou seu responsável ${ }^{66}$.

As NT e os AM fazem parte das informações proativas que um CIM desenvolve, devendo sempre seguir os critérios de qualidade e independência ${ }^{22}$, bem como devem atender a necessidades de informação recorrente ou temas de importância da mídia, que requeiram informação segura ${ }^{25}$, aspectos que foram contemplados para as NT e o AM desenvolvidos pelo trabalho colaborativo dos CIM neste estudo.
Em diagnóstico situacional dos CIM/SIM, membros da Rebracim em 2017 apontaram fragilidades dos serviços relacionadas à carência de recursos humanos, à dificuldade na aquisição de bases de dados e ao não reconhecimento dos gestores sobre a importância do serviço $0^{22}$. 0 trabalho colaborativo relatado aqui apresenta melhor aproveitamento dos recursos humanos, permitindo a troca de experiência, com compartilhamento das literaturas acessadas nas bases de dados de cada centro e maior qualidade da informação prestada, conduzindo a uma maior valorização dos serviços independentes, contribuindo com a importância dos serviços de informação como um todo, o que reforça a necessidade do trabalho colaborativo.

Não foi possível, entretanto, a participação de outros CIM que integram a Rebracim para viabilizar esse trabalho como uma atividade da rede em si. É necessário pensar estratégias que possibilitem uma maior participação da rede de SIM/CIM brasileiros, com o seu fortalecimento em todas as regiões do Brasil para produção de informações com o mais elevado nível de confiança e qualidade, ampliando o alcance nacional e até internacional.

\section{CONCLUSÕES}

As epidemias e pandemias colocam desafios para a comunidade internacional. A pandemia do novo coronavírus trouxe junto a infodemia, o que torna o papel dos CIM/SIM fundamental para a disseminação de informações seguras e confiáveis. A experiência do trabalho na elaboração das NT e do AM sobre o uso de medicamentos na COVID-19 demonstra que os CIM estão exercendo seu papel de maneira relevante no combate a esse grave problema de saúde pública. Acesso à informação de qualidade, baseada nas melhores evidências, é o melhor caminho e deve ser incentivado.

Verificou-se que é possível o trabalho a distância, em parceria, com ampliação da capacidade de produção dos serviços independentes, mas principalmente com potencialização da qualidade do trabalho desempenhado, ampliando a difusão e disseminação de informações voltadas à promoção do URM entre os profissionais de saúde, os gestores e a comunidade.

\section{REFERÊNCIAS}

1. World Health Organization - WHO. Coronavirus disease 2019 (COVID-19). Situation Report 51. 11 mar 2020[acesso 7 jul 2020]. Disponível em: https://www.who.int/docs/default-source/coronaviruse/ situation-reports/20200311-sitrep-51-COVID-19. pdf?sfvrsn=1ba62e57_10

2. Council for International Organizations of Medical Sciences - CIOMS. Medicines assessment during public health emergencies needs good science, best practices and proper communication. Geneva: Council for International Organizations of Medical Sciences; 2020[acesso 7 jul 2020]. Disponível em:
https://cioms.ch/wp-content/uploads/2020/06/CIOMS_ WGXII_Statement.pdf

3. Cañás $M$, Urtasun MA. La evidencia en tiempos de coronavirus (COVID-19). Evid Actual Pract Ambul. 2020;23(2):1-5.

4. University of Oxford. Low-cost dexamethasone reduces death by up to one third in hospitalised patients with severe respiratory complications of COVID-19. Recovery News. 16 jun 2020[acesso 7 jul 2020]. Disponível em: https://www.recoverytrial.net/ news/low-cost-dexamethasone-reduces-death-by-up-toone-third-in-hospitalised-patients-with-severe-respiratorycomplications-of-COVID-19 
5. National Institute of Allergy and Infectiour Diseases - NIAID. National institute of health clinical trial shows remdesivir accelerates recovery from advanced COVID-19. News \& Events. 29 abr 2020[acesso 7 jul 2020]. Disponível em: https://www.niaid.nih.gov/news-events/nih-clinical-trialshows-remdesivir-accelerates-recovery-advanced-COVID-19

6. Mota DM, Kuchenbecker RDS. Considerações sobre o uso de evidências científicas em tempos de pandemia: o caso da COVID-19. Vigil Sanit Debate. 2020;8(2):2-9. https://doi.org/10.22239/2317-269x.01541

7. Kalil AC. Treating COVID-19: off-label drug use, compassionate use, and randomized clinical trials during pandemics. JAMA. 2020;323(19):1897-8. https://doi.org/10.1001/jama.2020.4742

8. Caly L, Druce JD, Catton MG, Jans DA, Wagstaff KM. The FDA approved drug ivermectin inhibits the replication of SARS-COV-2 in vitro. Antiviral Res. 2020;178:3-6. https://doi.org/10.1016/j.antiviral.2020.104787

9. Liu M, Caputi TL, Dredze M, Kesselheim AS, Ayers JW. Internet searches for unproven COVID-19 therapies in the United States. JAMA Internal Medicine. 29 abr 2020.

10. Cinatl J, Michaelis M, Hoever G, Preiser W, Doerr HW. Development of antiviral therapy for severe acute respiratory syndrome. Antiviral Res. 2005;66(2-3):81-97. https://doi.org/10.1016/j.antiviral.2005.03.002

11. Sheahan TP, Sims AC, Leist SR, Schäfer A, Won J, Brown AJ et al. Comparative therapeutic efficacy of remdesivir and combination lopinavir, ritonavir, and interferon beta against MERS-COV. Nat Commun. 2020;11(1):1-14. https://doi.org/10.1038/s41467-019-13940-6

12. Agencia Española de Medicamentos y Productos Sanitarios - AEMPS. Cloroquina/hidroxicloroquina: precauciones y vigilancia de posibles reacciones adversas en pacientes con COVID-19. Acciones Informativas. 22 abr 2020[acesso 7 jul 2020]. Disponível em: https://www.aemps.gob.es/informa/ notasinformativas/medicamentosusohumano-3/seguridad1/2020-seguridad-1/cloroquina-hidroxicloroquinaprecauciones-y-vigilancia-de-posibles-reacciones-adversasen-pacientes-con-COVID-19/

13. Agence Nationale de Sécurité du Médicaments et des produits de Santé - ANSM. Médicaments utilisés chez les patients atteints du COVID-19: une surveillance renforcée des effets indésirables. Point d'Information. 10 abr 2020[acesso 7 jul 2020]. Disponível em: https://www.ansm.sante.fr/S-informer/ Points-d-information-Points-d-information/Medicamentsutilises-chez-les-patients-atteints-du-COVID-19-unesurveillance-renforcee-des-effets-indesirables-Point-dinformation-actualise-le-14-05-2020

14. European Medicines Agency - EMA. EU actions to support availability of medicines during COVID-19 pandemic: update 6. Press Release. 15 maio 2020[acesso 7 jul 2020]. Disponível em: https://www.ema.europa.eu/en/ documents/press-release/eu-actions-support-availabilitymedicines-during-COVID-19-pandemic-update-6_en.pdf

15. Agência Nacional de Vigilância Sanitária - Anvisa. Edital de chamamento $\mathrm{N}^{\circ}$, de 13 de março de 2020. Convoca empresas a fornecerem informações sobre produtos sujeitos à vigilância sanitária com risco de desabastecimento, utilizados ou não como insumos para enfrentamento do novo coronavírus (SARS-COV-2), de forma a identificar proativamente possíveis ameaças à saúde pública, devido às consequências relacionadas à pandemia de COVID-19. Diário Oficial União. 16 mar 2020.

16. Ministério da Saúde (BR). Diretrizes das atividades e estrutura dos centros de informação sobre medicamentos no SUS. Brasília: Ministério da Saúde; 2015[acesso 7 jul 2020]. Disponível em: https://www.saude.gov.br/images/ pdf/2017/maio/02/Diretrizes-das-Atividades-e-estruturados-Centros-de-Informacoes-Medicamentos-no-SUS.pdf

17. Centro Nacional de Documentación e Información de Medicamentos - CENADIM. Acerca de la repecim. Lima: Centro Nacional de Documentación e Información de Medicamentos; 2020[acesso 7 jul 2020]. Disponível em: http://bvcenadim.digemid.minsa.gob.pe/repecim

18. Red Argentina de Centros de Información sobre Medicamentos - RACIM. Disponibilidade de medicamentos com hidroxicloroquina: llamado a la solidaridad. Córdoba: Red Argentina de Centros de Información sobre Medicamentos; 2020[acesso 7 jul 2020]. Disponível em: https: / / www.fundacionfemeba.org. ar/blog/farmacologia-7/post/racim-disponibilidad-demedicamentos-con-hidroxicloroquina-llamado-a-lasolidaridad- 47678

19. Ministério da Saúde (BR). Portaria $N^{\circ} 2.647$, de 4 de novembro de 2013. Institui a rede brasileira de centros e serviços de informação sobre medicamentos (Rebracim). Diário Oficial União. 5 nov 2013.

20. National Health Service - NHS. UK medicines information: a briefing paper. London: National Health Service; 2010[acesso 7 jul 2020]. Disponível em: https://www.ukmi. nhs.uk/filestore/ukmiua/UKMibriefingpaper-final.pdf

21. Nova Manosalva MA, López Gutiérrez JJ, Cañas M. Drug information centers: an overview to the concept. Rev Colomb Cienc Quim Farm. 2016;45(2):243-55. https://doi.org/10.15446/rcciquifa.v45n2.59940

22. Ministério da Saúde (BR). Centros e serviços de informação sobre medicamentos: princípios, organização, prática e trabalho em redes para promoção do uso racional de medicamentos. Brasília: Ministério da Saúde; 2020.

23. Michaelis $\mathrm{H}$, Michaelis $\mathrm{C}$. Michaelis dicionário brasileiro da língua portuguesa. São Paulo: Melhoramentos; 2020[acesso 7 jul 2020]. Disponível em: http://michaelis.uol.com.br/moderno-portugues/

24. World Health Organization - WHO. Promoting rational use of medicines: core components. Who Policy Perspectives on Medicine. 2002[acesso 7 jul 2020]. Disponível em: http://archives.who.int/tbs/ rational/h3011e.pdf

25. Vidotti CCF, Hoefler R, Silva EV, Bergsten-Mendes G. Sistema brasileiro de informação sobre medicamentos Sismed. Cad Saude Publica. 2000;16(4):1121-6. https://doi.org/10.1590/S0102-311X2000000400030 
26. Centros de Informações sobre Medicamentos - CIM. Ausência de evidências científicas que relacionem o agravamento da infecção causada pelo novo coronavírus (SARS-COV-2) e a utilização de alguns medicamentos antihipertensivos e anti-inflamatórios não esteroidais (AINEs). Nota Técnica Informativa. 17 mar 2020[acesso 6 jul 2020]. Disponível em: http: //www.crf-ba.org.br/site/wp-content/ uploads/2020/03/Aus\%C3\%AAncia.pdf

27. Centros de Informações sobre Medicamentos - CIM. Evidências científicas frágeis sobre a utilização do medicamento hidroxicloroquina ou cloroquina no tratamento e/ou prevenção de infecção pelo novo coronavírus (SARS-COV-2). Nota Técnica Informativa 2. 7 abr 2020[acesso 6 jul 2020]. Disponível em: http://www.crf-ba.org.br/site/wp-content/ uploads/2020/04/Nota-te\%CC\%81cnica-n\%C2\%BO-2_hidroxicloroquina-e-COVID-19.pdf

28. Centros de Informações sobre Medicamentos - CIM. Evidências científicas sobre a utilização do medicamento ivermectina para tratamento de infecção pelo novo coronavírus (SARS-COV-2). Nota Técnica Informativa 3. 20 abr 2020[acesso 6 jul 2020]. Disponível em: http://www.crf-ba.org.br/site/wp-content/ uploads $/ 2020 / 04 /$ Nota-te\%CC\%81cnica-n\%C2\%BO-3_ivermectina-e-COVID-19.docx.pdf

29. Centros de Informações sobre Medicamentos - CIM. Análise de evidências científicas sobre a suplementação de vitamina $D$ (colecalciferol) como prevenção e tratamento da COVID-19. Nota Técnica Informativa 4. 8 maio 2020[acesso 6 jul 2020] Disponível em: http://www.crf-ba.org.br/site/wp-content/ uploads/2020/05/notatecnicavitaminad.pdf

30. Ministério da Saúde (BR). Diretrizes metodológicas: elaboração de pareceres técnico-científicos. 4a ed. Brasília: Ministério da Saúde; 2014.

31. Centros de Informações sobre Medicamentos - CIM. Risco de neurotoxicidade causada pela ivermectina no tratamento da COVID-19. Alerta. 11 jun 2020[acesso 6 jul 2020]. Disponível em: http://www.crf-ba.org.br/ site/wp-content/uploads/2020/06/ALERTA-N-01_2020Ivermectina-e-neurotoxicidade.pdf http://portais.univasf. edu.br/cim/cim/alertas/alerta-n-01_2020-ivermectina-eneurotoxicidade.pdf

32. Rios-Neto ELG. Pobreza, migrações e pandemias. In: Anais da $1{ }^{\text {a }}$ Conferência Nacional de Política Externa e Política Internacional; Rio de Janeiro, Brasil. Brasília: Fundação Alexandre de Gusmão; 2007.

33. Martínez EC, Borja-Terán B, Hernández-Rojas E, Velázquez-Zúñiga $C$. Síndrome agudo respiratorio severo (SARS). Med Crit. 2003;17(2):56-79.

34. Celly TAI, Villamil JLC. Estudio retrospectivo del nuevo coronavirus MERS-COV 2012-2013. Rev Lasallista Investig. 2014;11(2):71-7. https://doi.org/10.22507/rli.v11n2a8

35. Daraz L, Morrow AS, Ponce OJ, Beuschel B, Farah MH, Katabi A et al. Can patients trust online health information? A meta-narrative systematic review addressing the quality of health information on the internet. J Gen Intern Med. 2019;34(9):1884-91. https://doi.org/10.1007/s11606-019-05109-0

36. Pan American Health Organization - PAHO. Understanding the infodemic and misinformation in the fight against COVID-19. Factsheet 5. 2020[acesso 7 jul 2020]. Disponível em: https://iris.paho.org/bitstream/ handle/10665.2/52052/Factsheet-infodemic_eng. pdf?sequence $=5$

37. Zarocostas J. How to fight an infodemic. Lancet. 2020;395(10225):676. https://doi.org/10.1016/S0140-6736(20)30461-X

38. Palácio MAV, Takenami I. Em tempos de pandemia pela COVID-19: o desafio para a educação em saúde. Vigil Sanit Debate. 2020;8(2):10-5. https://doi.org/10.22239/2317-269x.01530

39. Associação de Medicina Intensiva Brasileira - AMIB. Diretrizes para o tratamento farmacológico da COVID-19. São Paulo: Associação de Medicina Intensiva Brasileira; 2020[acesso 7 jul 2020]. Disponível em: https://sbpt.org. $\mathrm{br} /$ portal/wp-content/uploads/2020/05/Diretrizes-para-oTratamento-Farmacologico-da-COVID-v18mai2020.pdf

40. Strina A, Teixeira MG, Costa MCN, Grassi MFR, Barreto ML. Ivermectina não deve ser indicada para tratamento de COVID-19. Rede Covida Nota Técnica 6. 2020[acesso 7 jul 2020]. Disponível em: http://analisepoliticaemsaude.org/oaps/documentos/ noticias/nota-ivermectina-nao-deve-ser-indicada-paratratamento-de-COVID-19/

41. Pan American Health Organization - PAHO. Recommendation regarding the use of ivermectin as a treatment for COVID-19. Washington: Pan American Health Organization; 2020[acesso 7 jul 2020]. Disponível em: https://iris. paho.org/handle/10665.2/52372

42. Sociedade Brasileira de Farmácia Clínica - SBFC. Posicionamento sobre o uso de ivermectina e nitazoxanida na COVID-19. Brasília: Sociedade Brasileira de Farmácia Clínica; 2020[acesso 7 jul 2020]. Disponível em: http: / /www.farmaciaclinica.org.br/ wp-content/uploads/2020/06/Posicionamento-da-SBFCivermectina-e-nitazoxanida-na-COVID-19.pdf

43. Red de Centros de Información de Medicamentos de Latinoamerica y el Caribe - Red CIMLAC. Tratamento farmacológico para COVID-19: actualización de la evidencia. Washington: Organizacíon Panamericana de la Salud; 2020[acesso 7 jul 2020]. Disponível em: https://web2.redcimlac.org/images/files/red\%20 cimlactratamientoscovidsegunda\%20partejunio.pdf

44. Ministerio de Salud (PE). Resolución ministerial $N^{\circ} 270$, de 8 de mayo de 2020. Prevención, diagnóstico y tratamiento de personas afectadas por COVID-19. Normas Legales. 9 maio 2020.

45. Ministerio de Salud (BO). Ministerio de salud autoriza uso de ivermectina contra el COVID-19 bajo protocolo. La Paz: Ministerio de Salud; 2020[acesso 7 jul 2020]. Disponível em: https://www.minsalud.gob.bo/4157ministerio-desalud-autoriza-uso-de-ivermectina-contra-elCOVID-19-bajo-protocolo 
46. Brito A. Distrito do Bailique recebe da prefeitura de Macapá kits de EPI, testes rápidos e medicação para combate à COVID-19. Prefeitura de Macapá Notícia. 2020[acesso 9 jun 2020]. Disponível em: http://macapa.ap.gov.br/ coronavirus/category/noticias/page/7/

47. Haôr V. Saúde: kits de medicamentos contra a COVID-19 começam a ser distribuídos à população. Ascom Prefeitura de Maraba. 22 maio 2020[acesso 9 jun 2020]. Disponível em: https://maraba.pa.gov.br/saude-kits-demedicamentoscontra-a-COVID-19-comecam-a-ser-distribuidos-a-populacao/

48. Prefeitura de Tapira. Unidades básicas de saúde recebem kits com ivermectina, azitromicina e sulfato de zinco. Notícias Saúde. 3 jun 2020[acesso 7 jul 2020]. Disponível em: https: / / www.tabira.pe.gov.br/informa.php?id=370

49. Unimed de Fortaleza Cooperativa de Trabalho Médico Unimed Fortaleza. Distribuição de medicamentos para tratamento da COVID-19. Notícias. 18 maio 2020[acesso 9 jun 2020]. Disponível em: https://www.unimedfortaleza.com.br/ distribuicao-de-medicamentos-para-tratamento-da-COVID-19

50. Universidade Federal do Rio Grande do Sul - UFRGS. Avaliação das evidências científicas sobre o uso de hidroxicloroquina/cloroquina como terapia específica para COVID-19. TelessaúdeRS. 22 maio 2020[acesso 7 jul 2020]. Disponível em: https://www.ufrgs.br/telessauders/ noticias/avaliacao-das-evidencias-cientificas-sobre-o-usode-hidroxicloroquina-cloroquina-como-terapia-especificapara-COVID-19/

51. Conselho Federal de Farmácia - CFF. Carta aberta do CFF aos farmacêuticos e à sociedade: em defesa da vida, da promoção e da proteção à saúde da população. Brasília: Conselho Federal de Farmácia; 2020[acesso 7 jul 2020]. Disponível em: http: / / www.cff.org.br/noticia.php?id=5788

52. Instituto de Gestão Estratégica de Saúde do Distrito Federal - IGES-DF. Uso de cloroquina e hidroxicloroquina para tratamento da COVID-19: qual é a evidência? Nota Técnica. 25 mar 2020[acesso 6 jul 2020]. Disponível em: https: / /igesdf.org.br/wp-content/uploads/2020/03/ Informativo-Cloroquina-e-Hidroxicloroquina-25_03_2020.pdf

53. Conselho Regional de Farmácia do Estado de São Paulo CRF-SP. CRF-SP emite nota sobre uso de cloroquina e outros medicamentos no tratamento de COVID-19. Nota Técnica. 18 maio 2020[acesso 6 jul 2020]. Disponível em: https:// www.crfsp.org.br/noticias/11299-nota-t\%C3\%A9cnica-2. html

54. Agência Nacional de Vigilância Sanitária - Anvisa. Nota técnica sobre cloroquina e hidroxicloroquina. Brasília: Agência Nacional de Vigilância Sanitária; 2020[acesso 20 mar 2020]. Disponível em: http://portal.anvisa.gov.br/documents/219201/4340788/ Nota+Te\%C2\%B4cnica+sobre+ Cloroquina+e+Hidr oxicloroquina.pdf/659d0105-60cf-4cab-b80a-fa0e29e2e799

55. Sociedade Brasileira de Infectologia - SBI. Nota de esclarecimento: uso de hidroxicloroquina para COVID-19. São Paulo: Sociedade Brasileira de Infectologia; 2020[acesso 7 jul 2020]. Disponível em: https://www.infectologia. org.br/admin/zcloud/125/2020/03/ c9b8d4f743ac6534 9e051b8638af7ee90d9a41f2f09a84f0ff83fc559fdf8b5f.pdf
56. Rede de Cooperação em Farmacologia do Centro-Norte-Nordeste Brasileiro. Carta aberta à sociedade brasileira sobre evidências científicas e o uso da hidroxicloroquina e cloroquina no manejo da pandemia por COVID-19 no Brasil. São Paulo: Sociedade Brasileira de Farmacologia e Terapêutica Experimental; 2020[acesso 7 jul 2020]. Disponível em: https://www.sbfte.org. $\mathrm{br} / 2020 / 05 / 26 /$

57. Fundação Oswaldo Cruz - Fiocruz. Orientações sobre o uso da cloroquina para tratamento de pacientes infectados com SARS-COV-2, agente etiológico da COVID-19. Rio de Janeiro: Fundação Oswaldo Cruz; 2020[acesso 7 jul 2020]. Disponível em: https://portal.fiocruz.br/sites/portal.fiocruz.br/ files/documentos/orientacoes_sobre_a_cloroquina_nota_ tecnica_.pdf

58. Sociedade Brasileira de Cardiologia - SBC. Infecção pelo coronavírus 2019 (COVID-19). SBC Informa. 13 mar 2020[acesso 7 jul 2020]. Disponível em: http://www. cardiol.br/sbcinforma/2020/20200313-comunicadocoronavirus.html

59. Ministério de Salud $(\mathrm{CH})$. Uso de ibuprofeno u otros Aines en personas con sospecha o confirmación de COVID-19. Santiago: Ministério de Salud; 2020[acesso 7 jul 2020]. Disponível em: https://diprece.minsal.cl/temas-desalud/temas-de-salud/guias-clinicas-no-ges/guiasclinicas-no-ges-enfermedades-transmisibles/COVID-19/ recomendaciones/

60. Ministério de Salud $(\mathrm{CH})$. Uso de IECA/ARA II en contexto de pandemia de COVID-19. Santiago: Ministério de Salud; 2020[acesso 7 jul 2020]. Disponível em: https://diprece. minsal.cl/temas-de-salud/temas-de-salud/guias-clinicasno-ges/guias-clinicas-no-ges-enfermedades-transmisibles/ COVID-19/recomendaciones/

61. World Health Organization - WHO. The use of non-steroidal anti-inflammatory drugs (Nsaids) in patients with COVID-19. Newsroom. 19 abr 2020[acesso 7 jul 2020]. Disponível em: https://www.who.int/news-room/commentaries/detail/ the-use-of-non-steroidal-anti-inflammatory-drugs-(nsaids)in-patients-with-COVID-19

62. Conselho Regional de Medicina do Estado do Rio Grande do Sul - CREMERS. Nota técnica do Cremers sobre a utilização da suplementação de vitamina $D$ para prevenção e/ou tratamento de infecções por COVID-19. Porto Alegre: Conselho Regional de Medicina do Estado do Rio Grande do Sul; 2020[acesso 7 jul 2020]. Disponível em: https://cremers.org. $\mathrm{br} / \mathrm{wp}$-content/uploads/2020/05/11.05.2020-Notat\%C3\%A9cnica-do-CREMERS-sobre-a-utiliza\%C3\%A7\%C3\%A3oda-suplementa\%C3\%A7\%C3\%A3o-de-vitamina-Dpara-preven\%C3\%A7\%C3\%A3o-eou-tratamento-deinfec\%C3\%A7\%C3\%B5es-por-COVID-19.pdf

63. Ministério da Saúde (BR). Vitamina D na prevenção e tratamento de pacientes com COVID-19. Brasília: Ministério da Saúde; 2020[acesso 7 jul 2020]. Disponível em: https://portalarquivos.saude.gov. $\mathrm{br} /$ images/pdf/2020/May/13/VitaminaD-COVID19atualizacaoA.pdf 
64. Sociedade Brasileira de Endocrinologia e Metabologia SBEM. Nota de esclarecimento: vitamina D e COVID-19. Rio de Janeiro: Sociedade Brasileira de Endocrinologia e Metabologia; 2020[acesso 7 jul 2020]. Disponível em: https://www.endocrino.org.br/media/posicionamento_ sbem_e_abrasso_-_vitamina_d_e_COVID-19_final_(1).pdf

65. Sociedade Brasileira de Dermatologia - SBD.

COVID-19: há falta de evidências científicas sobre a prevenção por meio da exposição ao sol ou ao consumo de vitamina D. Rio de Janeiro: Sociedade Brasileira de Dermatologia; 2020[acesso 7 jul 2020]. Disponível em: https: / / www.sbd.org.br/mm/cms/2020/04/12/nota-sbdvitamina-covid-final.pdf

66. Ministério Público do Pará - MP-PA. Recomendação $\mathrm{N}^{\circ} 11$, de 11 de maio de 2020. Diário Oficial do Estado. 12 maio 2020.

\section{Contribuição dos autores}

Brandão MFBO, Passos ACB, Unfer TC, Nunes DM - Concepção, planejamento (desenho do estudo), aquisição, análise, interpretação dos dados e redação do trabalho. Matos LEO, Soares LP, Pio IDSL, Monteiro MP - Análise, redação do trabalho. Todos os autores aprovaram a versão final do trabalho.

Conflito de Interesse

Os autores informam não haver qualquer potencial conflito de interesse com pares e instituições, políticos ou financeiros deste estudo.

Esta publicação está sob a licença Creative Commons Atribuição 3.0 não Adaptada.

Para ver uma cópia desta licença, visite http://creativecommons.org/licenses/by/3.0/deed.pt_BR. 